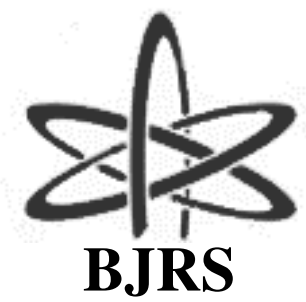

BRAZILIAN JOURNAL

$\mathrm{OF}$

RADIATION SCIENCES

03-1A (2015) 01-10

\title{
Teste de aceite de um activímetro a ser usado como referência na implantação de uma nova metodologia de calibração
}

\author{
E. L. Corrêa $a^{\text {a }}$ L. T. Kuahara ${ }^{\text {a }}$ M. P. A. Potiens ${ }^{a}$ \\ ${ }^{a}$ Gerência de Metrologia das Radiações, Instituto de Pesquisas Energéticas e Nucleares, 05508-000,São Paulo-SP, \\ Brasil \\ eduardo.correa@usp.br
}

\begin{abstract}
RESUMO
A medicina nuclear é a área da física médica que utiliza radioisótopos para realização de exames diagnósticos. Esses elementos são administrados no paciente e a radiação emitida por eles é detectada por um equipamento que faz uma varredura de seu corpo, e a imagem é construída por um programa de computador. Para entrar em operação, um serviço de medicina nuclear deve ter, dentre outros equipamentos de trabalho e segurança, equipamentos de medição de radiação devidamente calibrados. Porém não existe, no Brasil, um programa de calibração de ativímetros, o que faz com que acabem ocorrendo muitas incertezas nas leituras desses instrumentos. O objetivo deste trabalho é apresentar os resultados de um teste de aceite realizado em um ativímetro recém-adquirido pelo IPEN, para ser utilizado como referência na implantação de uma nova metodologia de calibração desses equipamentos. Foi verificado o comportamento de um ativímetro da Capintec, modelo CRC-25R, utilizando três fontes de controle $\left({ }^{137} \mathrm{Cs},{ }^{57} \mathrm{Co},{ }^{133} \mathrm{Ba}\right)$. Os testes foram baseados na norma CNEN-NN 3.05, no código de prática TRS-454 e no manual do fabricante e incluem: inspeção física, voltagem da câmara, auto zero, verificação da radiação de fundo, data check e repetibilidade. Os testes de linearidade e geometria não puderam ser realizados, pois o laboratório no qual o ativímetro se encontra ainda não possui autorização para receber fontes não-seladas ( ${ }^{99 \mathrm{~m}} \mathrm{Tc}$ ). Em todos os testes os resultados obtidos estavam dentro das exigências da norma da CNEN. O equipamento já foi colocado em funcionamento e passa periodicamente pelos testes de controle de qualidade.
\end{abstract}

Keywords: Nuclear Medicine, Instrumentation, Radioisotopes

Palavras-chave: Medicina Nuclear, Instrumentação, Radioisótopos. 


\section{INTRODUÇÃO}

A medicina nuclear é uma área da física médica que utiliza radioisótopos no diagnóstico e tratamento de doenças [1]. As aplicações de radioisótopos na medicina nuclear são muitas, e nesse caso são normalmente utilizados para analisar a fisiologia do tecido a ser estudado [2].

No Brasil, para entrar em operação, um serviço de medicina nuclear deve ser autorizado pela Agência Nacional de Vigilância Sanitária (ANVISA) e pela Comissão Nacional de Energia Nuclear (CNEN), que solicitam toda a documentação necessária, tais como certificados de calibração dos monitores de área e de superfície, contrato com um supervisor de medicina nuclear entre outros $[3,4,5]$.

O medidor de atividade (ativímetro) é um dos equipamentos essenciais a serem adquiridos por um serviço de medicina nuclear [5]. Ele é utilizado para verificar se a atividade de um dado radiofármaco é adequada para ser administrada no paciente.

Porém, apesar de sua importância nessa área, a calibração dos medidores de atividade não é requisitada pelas autoridades brasileiras, e não existe um laboratório que forneça esse serviço.

O Laboratório de Calibração de Instrumentos (LCI) do Instituto de Pesquisas Energéticas e Nucleares (IPEN) possui, para fins de calibração e pesquisa, as qualidades de radiodiagnóstico implantadas em seu sistema de raios-X [6,7,8], e agora está sendo implementada uma metodologia de calibração de medidores de atividade.

O objetivo do presente estudo é apresentar os resultados obtidos durante os testes de aceite de um novo medidor de atividade adquirido pelo LCI e que será usado como referência na implantação dessa metodologia.

\section{MATERIAIS E MÉTODOS}

\subsection{Materiais}

O teste de aceite foi realizado em um medidor de atividade Capintec, modelo CRC-25R (Figura 1), usando três fontes de controle $\left({ }^{137} \mathrm{Cs},{ }^{57} \mathrm{Co},{ }^{133} \mathrm{Ba}\right.$, conforme Figura 2). 
Figura 1. Medidor de atividade Capintec, modelo CRC-25R.

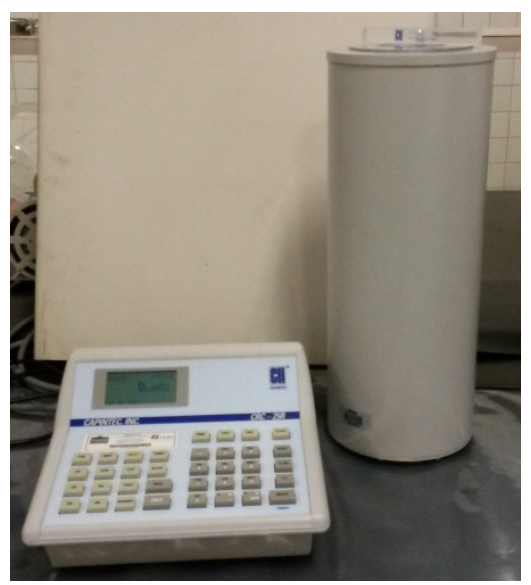

Figure 2. Da esquerda para a direita: fontes usadas no controle de qualidade ${ }^{57} \mathrm{Co},{ }^{137} \mathrm{Cs}$ e ${ }^{133} \mathrm{Ba}$

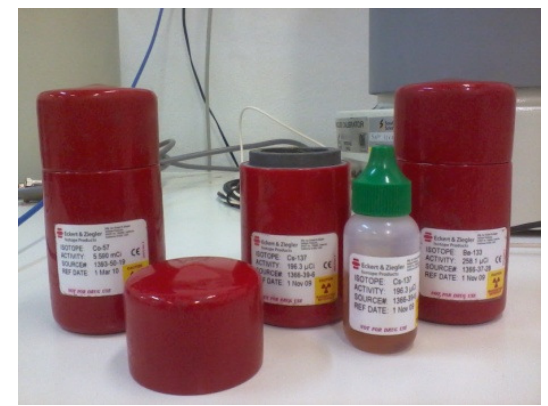

Na Tabela 1 são apresentadas as principais características desses radioisótopos.

Tabela 1. Características principais dos radioisótopos usados no teste de aceite

\begin{tabular}{ccccc}
\hline Radioisótopo & $\begin{array}{c}\text { Energia do fóton } \\
\text { principal }(\mathbf{k e V})\end{array}$ & Meia-vida & $\begin{array}{c}\text { Atividade } \\
\text { nominal }(\boldsymbol{\mu C i})\end{array}$ & $\begin{array}{c}\text { Data de } \\
\text { referência }\end{array}$ \\
\hline${ }^{\mathbf{1 3 7}} \mathbf{C s}$ & 662 & 30,0 anos & 196,3 & 01 Nov 2009 \\
${ }^{\mathbf{5 7}} \mathbf{C o}$ & 122 & 271 dias & 5590 & 01 Março 2010 \\
${ }^{\mathbf{1 3 3}} \mathbf{B a}$ & $81 ; 356$ & 10,7 anos & 258,1 & 01 Nov 2009 \\
\hline
\end{tabular}




\subsection{Métodos}

Os testes foram feitos tomando como base as informações obtidas na norma CNEN-NN 3.05 [5], no TRS-454 [9], no TECDOC-602 [10] e no manual do fabricante. Alguns testes foram chamados de "testes automáticos", pois esses testes são realizados automaticamente pelo equipamento ao acionar a opção "Daily Test" (teste diário).

\subsubsection{Inspeção Física}

O propósito desse teste é verificar as condições físicas do medidor de atividade, verificando se não existem danos físicos nos componentes, teclas, conectores, acessórios entre outros [10].

Esse procedimento deve ser realizado no momento da entrega do equipamento pelo fabricante, e o mesmo deve ser informado de qualquer dano encontrado [10].

\subsubsection{Tensão na Câmara (Teste Automático)}

Esse teste tem como objetivo verificar se a tensão aplicada na câmara é constante e está de acordo com o valor apresentado no manual do fabricante. Ele é realizado automaticamente pelo próprio sistema do medidor de atividade, e a mensagem "OK" aparece na tela se o valor estiver dentro do especificado.

\subsubsection{Radiação de Fundo (Teste Automático)}

De acordo com o TRS-454, esse teste é usado como um indicador de que o ruído eletrônico não está se deteriorando, e também que fontes externas de radiação não estejam presentes no local onde a medição será realizada [9].

Para a realização desse teste todas as fontes radioativas devem ser retiradas das proximidades do medidor de atividade. O resultado é então armazenado juntamente com o modelo e o número de série do equipamento, na data de realização do mesmo. Caso seja possível realizar um ajuste de zero no medidor de atividade, então se deve fazê-lo [9,10]. 


\subsubsection{Ajuste de Zero (Teste Automático)}

Em alguns medidores de atividade o ajuste de zero é feito automaticamente, e é parte da rotina dos testes diários. Esse é o caso do CRC-25R. Após a medição da radiação de fundo o equipamento realiza esse ajuste, tornando desnecessário o ajuste manual. Se o procedimento for feito corretamente a mensagem “OK” aparecerá na tela.

\subsubsection{Data Check (Teste Automático)}

Nesse teste o medidor de atividade faz uma verificação de todas as informações sobre as fontes inseridas no seu banco de dados. Após essa verificação, caso tudo esteja correto, a mensagem “OK” aparecerá no mostrador.

\subsubsection{Exatidão}

Exatidão de uma medição é definida como o grau de concordância entre um valor medido e um valor verdadeiro de um mensurando [11]. Para obter a exatidão $D$ a atividade medida $A_{i}$ no equipamento foi comparada com a atividade nominal $A_{v}$, corrigida para o decaimento, utilizando para isso a equação 1 [5]:

$$
D=\frac{\left(1-A_{i}\right)}{A_{v}} \times 100 \%
$$

\subsubsection{Precisão}

Precisão de uma medição é definida como o grau de concordância entre indicações ou valores medidos, obtidos por medições repetidas, no mesmo objeto ou em objetos similares, sob condições especificadas [11]. A precisão $P$ é obtida utilizando a equação 2:

$$
P=\frac{\left(A_{i}-\bar{A}\right)}{\bar{A}} \times 100 \%
$$


onde $A i$ é a medição da atividade e $\overline{\mathrm{A}}$ é a média das atividades medidas [5].

Para a obtenção da exatidão e precisão é necessário que algumas informações importantes sobre os radioisótopos, tais como tipo, atividade nominal e data de referência, sejam inseridas na memória do medidor de atividade.

\section{RESULTADOS E DISCUSSÕES}

A seguir estão os resultados obtidos durante os testes. Para facilitar na compreensão os testes automáticos (tensão na câmara, ajuste de zero e data check) são apresentados juntos.

\subsection{Inspeção Física e Testes Automáticos}

Não foi encontrado nenhum dano estrutural durante a inspeção física. Todos os conectores, botões e controles estavam funcionando adequadamente.

Durante o primeiro teste diário o ativímetro apresentou um bom comportamento, como apresentado na Tabela 2.

Tabela 2. Resultado obtido durante os "testes automáticos".

No visor é apresentada a mensagem "OK" quando o instrumento passa no teste

\begin{tabular}{cc}
\hline Teste & Status do Ativímetro \\
\hline Ajuste de Zero & $\mathrm{OK}$ \\
Radiação de Fundo & $\mathrm{OK}$ \\
Tensão na Câmara & $\mathrm{OK}$ \\
Data Check & $\mathrm{OK}$ \\
\hline
\end{tabular}

$\mathrm{O}$ ativímetro $\mathrm{CRC}-25 \mathrm{R}$ passou nos testes. $\mathrm{O}$ instrumento mediu uma atividade de radiação de fundo de aproximadamente $0,25 \mu \mathrm{Ci}$, muito abaixo do valor apresentado pelas fontes de referência. 


\subsection{Testes de Exatidão e Precisão}

Os resultados para a exatidão de medição são mostrados na Tabela 3.

Tabela 3. Resultados para a exatidão de medição. A variação entre o valor medido e a atividade nominal esteve dentro dos limites, em todos os casos.

\begin{tabular}{cccc}
\hline Radioisótopo & $\begin{array}{c}\text { Atividade Nominal } \\
(\boldsymbol{\mu C i})\end{array}$ & $\begin{array}{c}\text { Atividade Medida } \\
(\boldsymbol{\mu C i})\end{array}$ & $\begin{array}{c}\text { Variação } \\
(\boldsymbol{\%})\end{array}$ \\
\hline${ }^{\mathbf{1 3 7}} \mathbf{C s}$ & 179,6 & $185 \pm 2$ & $2,92 \pm 0,03$ \\
${ }^{57} \mathbf{C o}$ & 213 & $209 \pm 2$ & $-1,91 \pm 0,02$ \\
${ }^{\mathbf{1 3 3}} \mathbf{B a}$ & 200 & $201 \pm 2$ & $0,50 \pm 0,01$ \\
\hline
\end{tabular}

Os resultados para a precisão de medição apresentaram desvio padrão igual a zero, para os três radioisótopos utilizados, o que pode indicar tanto que esse instrumento é bastante estável quanto que ele não possui resolução suficiente para apresentar mais casas decimais em cada medição realizada. Isso será verificado ao longo do tempo, durante os testes diários, que serão realizadas de agora em diante.

\section{CONCLUSÕES}

Os testes de aceite foram realizados em um ativímetro Capintec, modelo CRC-25R, e apresentou resultados bastante satisfatórios. Nenhum dano foi encontrado na inspeção física. Durante os testes de tensão aplicada na câmara, radiação de fundo, ajuste de zero e data check a mensagem "OK" foi mostrada no visor do instrumento, o que indica que o mesmo estava funcionando adequadamente.

No teste de exatidão de medição foi obtida uma variação máxima de 2,29\%, o que está dentro dos limites apresentados por normas nacionais e internacionais. 
No teste de precisão de medição foi obtido um desvio padrão igual a zero, para os três radioisótopos utilizados, o que pode indicar tanto que esse instrumento é bastante estável quanto que ele não possui resolução suficiente para apresentar mais casas decimais em cada medição realizada. Uma verificação desse comportamento será feita durante a realização dos testes diários.

Em geral este instrumento apresentou características muito boas e uma grande estabilidade, e poderá ser utilizado como referência na implementação de uma nova metodologia de calibração de ativímetros.

\section{AGRADECIMENTO}

Os autores agradecem o suporte financeiro parcial da Fundação de Amparo à Pesquisa do Estado de São Paulo (FAPESP), Conselho Nacional de Desenvolvimento Científico e Tecnológico (CNPq), Coordenação de Aperfeiçoamento de Pessoal de Nível Superior (CAPES) e Ministério da Ciência e Tecnologia (MCT, Projeto: Instituto Nacional de Ciência e Tecnologia (INCT) em Metrologia das Radiações na Medicina), Brasil. 


\section{REFERÊNCIAS}

1. A. Gottschalk, E. J. Potchen, Diagnostic Nuclear Medicine, Williams and Wilkins, Baltimore, USA (1976).

2. A. F. G. da ROCHA, Medicina Nuclear. Ed. Guanabara Koogan, Rio de Janeiro, Brasil (1976).

3. CNEN - Comissão Nacional de Energia Nuclear. Licenciamento de Instalações Radiativas. CNEN-NN-6.02, Rio de Janeiro, Brasil, 2014.

4. ANVISA - Agência Nacional de Vigilância Sanitária. Diretrizes de Proteção Radiológica em Radiodiagnóstico Médico e Odontológico. Portaria 453, Brasil, 1998.

5. CNEN - Comissão Nacional de Energia Nuclear. Requisitos de Segurança e Proteção Radiológica para Serviços de Medicina Nuclear. CNEN-NN-3.05, Rio de Janeiro, Brasil, 2013.

6. E. L. Correa, V. Vivolo, M. P. A. Potiens, Quality control methodology and implementation of X-radiation standards beams, mammography level, following the standard IEC 61267, Applied Radiation and Isotopes, vol. 70, pp. 1396-1399, 2012.

7. P. C. Franciscatto, Caracterização das qualidades de radiação $X$ seguindo as recomendações da norma IEC 61267 no laboratório de calibração do IPEN, Dissertação de Mestrado, Instituto de Pesquisas Energéticas e Nucleares, Universidade de São Paulo, São Paulo, Brasil, 2009.

8. D. M. Dias, Estabelecimento de um novo método de calibração de câmaras de ionização tipo lápis para dosimetria em feixes de tomografia computadorizada, Dissertação de 
Mestrado, Instituto de Pesquisas Energéticas e Nucleares, Universidade de São Paulo, São Paulo, Brasil, 2010.

9. IAEA - International Atomic Energy Agency. Quality Assurance for Radioactivity Measurement in Nuclear Medicine. Technical Report Series No. 454, Vienna, 2007.

10. IAEA - International Atomic Energy Agency. Quality Control of Nuclear Medicine Instruments. IAEA-TEC-DOC-602, Vienna, 2007.

11. INMETRO - Instituto Nacional de Metrologia, Qualidade e Tecnologia. Vocabulário Internacional de Metrologia. 1 ${ }^{a}$ edição Luso-Brasileira do VIM 2012, Brasil, 2012. 